BMJ Paediatrics Open

\title{
Understanding COVID-19: are children the key?
}

\author{
Suz Warner (D) , Alex Richter, ${ }^{1}$ Zania Stamataki, ${ }^{1}$ Deirdre Kelly ${ }^{2}$
}

To cite: Warner $S$, Richter A, Stamataki Z, et al. Understanding COVID-19: are children the key? BMJ Paediatrics Open 2021;5:e001063. doi:10.1136/ bmjpo-2021-001063

Received 15 February 2021

Revised 23 April 2021

Accepted 1 May 2021
Check for updates

(c) Author(s) (or their employer(s)) 2021. Re-use permitted under CC BY-NC. No commercial re-use. See rights and permissions. Published by BMJ.

${ }^{1}$ Institute of Immunology and Immunotherapy, University of Birmingham, Birmingham, UK ${ }^{2}$ The Liver Unit, Birmingham Women's and Children's NHS Foundation Trust, Birmingham, UK

\section{Correspondence to} Dr Suz Warner; S.Warner@ bham.ac.uk

\section{ABSTRACT}

The devastating impact of the COVID-19 pandemic on global health and economic stability is immeasurable. The situation is dynamic and fast-evolving, with the world facing new variants of concern which may have immune escape potential. With threatened treatment and preventative strategies at stake, and the prospect of reinfection prolonging the pandemic, it is more crucial than ever to understand the pathogenesis of SARSCoV-2 infection, which intriguingly disproportionately affects adults and the elderly. Children infected with SARS-CoV-2 remain largely asymptomatic or undergo a transient mild illness. Understanding why children have a milder phenotype and a significant survival advantage may help identify modifiable risk factors in adults. Current evidence suggests adults with COVID-19 show variability in innate and adaptive immune responses, which result in uncontrolled proinflammatory cytokine production in some patients, leading to severe disease and mortality. Children with acute COVID-19 infection seldom progress to acute respiratory distress syndrome and are less likely to exhibit the cytokine storm which is so prominent in adults. Even with the Kawasaki-like illness, a hyperinflammation syndrome also known as paediatric multisystem inflammatory syndrome temporally associated with SARS-CoV-2, mortality is low. The key to successfully combating SARS-CoV-2 and future zoonotic pandemics may lie in understanding these critical differences and merits focused consideration and research. The impact of community transmission among asymptomatic children is unknown; sustained global decline in infection rates and control of the COVID-19 pandemic may not be achieved until vaccination of children occurs. In this review, we discuss the fundamental differences in the immune response between children and adults in the fight against SARS-CoV-2.

\section{INTRODUCTION}

The COVID-19 pandemic has had a catastrophic impact on world health and economic stability. It is hoped that social normalcy and economic restoration will return following global SARS-CoV-2 vaccination. The emergence of mutant strains raises concerns over the efficacy of recently licensed vaccines, questioning the degree of cross-immunity and protection afforded by these vaccines. Whether the programmes are successful or not, we must continue to decipher and understand the pathogenesis of this

\section{Key messages}

- Significant differences exist in the immune response to SARS-CoV-2 between children and adults; understanding the basis for this variance may be crucial to controlling the pandemic.

- Emerging SARS-CoV-2 variants are more contagious and virulent and the role children have on community transmission of these new variants of concern needs to be established.

- Sustained control of the pandemic may not be achieved until children are included in the global vaccination against SARS-CoV-2.

devastating infection which disproportionately affects adults and the elderly.

Children infected with SARS-CoV-2 are either asymptomatic or experience a milder phenotype compared with adults. ${ }^{1}{ }^{2}$ This trajectory is similar to that experienced in the SARS-CoV-1 and Middle East respiratory syndrome (MERS) epidemics of 2002 and 2012. ${ }^{2}$ Children may experience fever and cough with COVID-19 but a loss of sense of smell or taste are less frequently encountered. $^{12}$

Pneumonitis progressing to acute respiratory distress syndrome (ARDS) and respiratory failure occurs in up to $19.5 \%$ of adult patients with COVID-19, with case fatality rates of $5 \%-8 \%$, rising to $14.8 \%$ in elderly patients $>80$ years. ${ }^{34}$ Children are quoted to account for $1 \%-5 \%$ of positive SARS-CoV-2 PCR tests, but this is likely to be under-represented as testing is skewed towards those who are symptomatic or require hospitalisation. ${ }^{56}$ Of the children who test positive, severe illness accounts for $1 \%-6 \%$, with $65 \%$ of these developing the Kawasaki-like illness, paediatric multisystem inflammatory syndrome temporally associated with SARS-CoV-2 (PIMS-TS), also known as multisystem inflammatory syndrome in children, but even then, mortality is low. ${ }^{7}$

Despite the milder phenotype, the pandemic has had a detrimental effect on child and adolescent health and education worldwide. ${ }^{8}$ Healthcare access and delivery 
were disrupted, with essential services like routine immunisations being postponed. ${ }^{8} 9$ Children are also considered at low risk of COVID-19 and are therefore not a priority in vaccination trials.

A literature review was performed using the online database PubMed to summarise the available information on the differences and similarities in SARS-CoV-2 infection between children and adults, with focus on transmission, immune response and immunopathogenesis. Clinical syndromes will be referred to as adult COVID-19, paediatric COVID-19 and PIMS-TS.

\section{TRANSMISSION}

SARS-CoV-2 transmits via aerosol and droplet spread but the virus is also detected in stool samples. ${ }^{10}$ Children experience more gastrointestinal symptoms of diarrhoea and vomiting than adults. Faecal-oral transmission nevertheless is not a common route of infection spread. ${ }^{10}$

The role of children in SARS-CoV-2 transmission is considered small and reflective of levels of transmission in their community. ${ }^{11}$

School and childcare centre COVID-19 outbreaks are not common especially if hand hygiene, wearing face masks (among teaching staff) and social distancing rules are followed, ${ }^{10} 11$ yet in the absence of testing asymptomatic individuals we are unable to track virus circulation in these settings. To date, contact tracing studies show $71 \%-90 \%$ of childhood SARS-CoV-2 infections were identified following infection of a family member, ${ }^{11}$ so we are probably underestimating the proportion of asymptomatic infected children.

With the fall of the reproduction number $(\mathrm{R})$ following the third national lockdown and success of the UK vaccination roll-out, schools reopened in March 2021 with pupils returning to face-to-face education. Cases of children testing positive for SARS-CoV-2 have since risen in the same way as observed following the reopening of schools in September $2020 .^{12}$ These observations suggest that children and young adolescents are likely to transmit SARS-CoV-2 within the school setting, their household and the general population, strengthening the need to include them in vaccination programmes.

Clinical trials testing the efficacy of approved SARS-CoV-2 vaccines in children have commenced, but programmes are currently paused until reports of rare clotting incidents in vaccinated young adults are fully investigated.

\section{CHILDHOOD AND ADULT IMMUNE RESPONSES TO SARS-COV-2}

Host innate and adaptive immune interactions are paramount in the clearance of viral infections. Innate immune cells like circulating neutrophils and monocytes express pattern recognition receptors (PRRs), for example, Tolllike receptors (TLR), which recognise 'danger signals' from microbial and endogenous molecules referred to as pathogen-associated and damage-associated molecular patterns, respectively. ${ }^{13}$ Innate cell activation occurs once these ligands bind to cell surface PRRs, leading to rapid proinflammatory cytokine production, immune cell recruitment and resultant tissue inflammation. TLRs are present on antigen-presenting cells (APCs) such as dendritic cells (DCs), monocytes and B lymphocytes. The spike glycoprotein has a strong affinity to TLR4; TLR4 binding and activation are seen in SARS-CoV-1 and SARS-CoV-2 infection. ${ }^{14}$ Moreover, APCs crucially link the innate and adaptive arms of the immune systems via the presentation of major histocompatibility complex (MHC) proteins to induce $\mathrm{T}$ lymphocyte activation; MHC class I present antigen peptides to cytotoxic CD8 and MHC class II to helper CD4 T cells. ${ }^{15}$ Cytotoxic CD8 cells kill virus-infected cells with perforin and granzyme release in addition to other proinflammatory cytokines. ${ }^{13} 15$ This T cell-mediated immunity combined with humoral immunity form the adaptive arm of the immune system and are discussed below in relation to SARS-CoV-2.

Adults with severe and critical COVID-19 display a suppressed adaptive arm in combination with a hyperactivated innate response, leading to uncontrolled proinflammatory cytokine production, extensive immune-mediated lung injury and multiorgan failure. ${ }^{1516}$

Pneumonitis and ARDS are rarely seen in paediatric COVID-19. ${ }^{16}{ }^{17}$ A hyperinflammation element is however part of the immunophenotype observed in PIMS-TS, a condition which characteristically occurs in children who are SARS-CoV-2 PCR negative and seropositive. ${ }^{18}$

\section{Innate immunity}

A lack of control of the primary SARS-CoV-2 infection and fatal COVID-19 has been strongly associated with hyperacute but ineffective innate immunity in adults. ${ }^{15} 16$

High numbers of circulating monocytes and neutrophils are found in severe adult COVID-19. Classical (CD14+CD16-) and intermediate (CD14+CD16+) monocytes, identified by fluorescence-activated cell sorting analysis (FACS), produce the inflammatory cytokines seen in severe COVID-19. ${ }^{16}$ These activated innate cells are rarely found in asymptomatic individuals or those with mild disease. Interestingly, non-classic monocytes (CD14- CD16+), which are anti-inflammatory and have a role in maintaining endothelial integrity, are reduced in number in severe adult COVID-19 (figure 1). ${ }^{16} 17$

Aside from phagocytosis, activated neutrophils form neutrophil extracellular traps (NETS), which are aptly named net-like structures made up mostly of chromatin and extracellular proteins that 'trap' microbes and infected cells. These NETS can prime the clotting cascade. NETS have thus been implicated in the microangiopathy and thrombosis seen in COVID-19. ${ }^{17}$ Children with PIMS-TS have endothelial cell damage and microthrombosis, especially seen in their skin lesions. ${ }^{18}$ The underlying microvascular and thrombotic pathogenesis may be shared in severe adult COVID-19 and PIMS-TS.

Natural killer (NK) cells are of lymphocyte lineage; however, they are considered part of the innate immune 


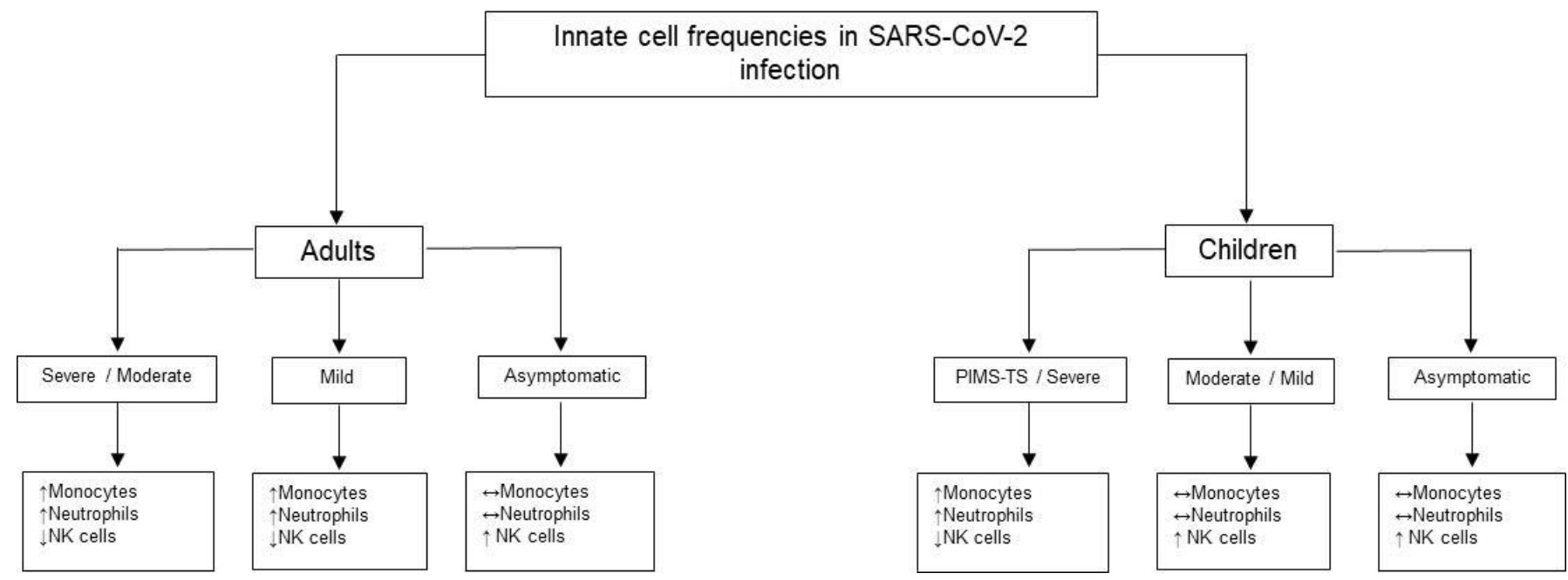

Figure 1 Innate cell frequencies in SARS-CoV-2 infection. ${ }^{15-18} \uparrow$, increased; $\downarrow$, decreased; $\leftrightarrow$, unchanged. NK cells, natural killer cells; PIMS-TS, paediatric multisystem inflammatory syndrome temporally associated with SARS-CoV-2.

system with their rapid ability to kill virus-infected cells by perforin and granzyme degranulation. NK cells are consistently reduced in COVID-19, but this is most marked in severe disease. ${ }^{16}$ Lower numbers of perforin-producing NK cells were found in intensive care unit (ICU) patients with COVID-19 compared with non-ICU patients in one study. ${ }^{19}$ This implies that NK cells are not only reduced in number but are also functionally impaired in severe disease. NK cell numbers are preserved in paediatric COVID-19. FACS analysis of peripheral blood shows similar reductions in NK cells and non-classic monocytes in children with PIMS-TS. ${ }^{20}$

Another theory for why children are more resilient to COVID-19 is trained immunity, the functional reprogramming of innate immune cells following common childhood infections and vaccinations, enabling memory cell formation which was previously considered unique to the adaptive immune system. ${ }^{126}$ This heterogeneous immunity is described with the BCG vaccination ${ }^{21}$ and is speculated to occur with coinfections; $10 \%$ of coinfections occur in children testing positive for SARS-CoV-2. ${ }^{21}$

APCs like DCs are suppressed in SARS-CoV-2, which indirectly downregulates $\mathrm{T}$ cell activation and function. ${ }^{17}$ DCs produce interferon (IFN) I and III, which are powerful antiviral cytokines important in early viral responses. SARS-CoV-2, like SARS-CoV-1 and MERS, are capable of immune escape by IFN inhibition, ${ }^{17}$ thereby eluding one of the host's most effective antiviral measures. One study found $10 \%$ of adults with life-threatening COVID-19 had autoantibodies directed against IFN I, reinforcing the significance of IFN and the detrimental effect of select immune deficiencies. ${ }^{22}$

\section{Adaptive immunity}

Lymphopaenia and lymphocyte exhaustion

Lymphopaenia and the high neutrophil to lymphocyte ratio described in severe adult COVID-19 are rarely observed in children. ${ }^{2324}$ In adults, CD4+ and CD8+ naïve and memory $\mathrm{T}$ cell numbers are reduced. ${ }^{25}$ Conversely, lymphocyte counts are preserved in paediatric COVID-19 but reduced in PIMS-TS. ${ }^{6} 20$

Adult patients with mild COVID-19 have clonally expanded CD8+ T lymphocytes. ${ }^{25}$ Moreover, individuals with mild disease were shown to mount a stronger SARSCoV-2-specific T cell response compared with those with severe disease, implying preserved adaptive immunity corresponds with superior disease control and recovery (figure 2). ${ }^{1725}$

In addition, $\mathrm{T}$ lymphocytes from patients with severe COVID-19 have higher expression of the cell exhaustion and inhibition markers Programmed cell death protein 1 (PD-1), Lymphocyte-activation gene 3 (LAG-3) and T-cell immunoglobulin and mucin-domain containing-3 (TIM3). ${ }^{24} 1724 \mathrm{~T}$ cell exhaustion correlates with impaired effector function. These findings suggest cellular immunity is affected both quantitatively and functionally.

$\mathrm{T}$ cell function and survival are a potential novel area of research in children with COVID-19; findings will contribute to our understanding of the age-related $\mathrm{T}$ cell response and behaviour in COVID-19.

\section{Humoral response}

Adult patients with COVID-19 mount a strong B cell response; neutralising antibodies against the spike glycoprotein are detected in the majority of convalescent patients with COVID-19. ${ }^{17}$ Although total B cell numbers are reduced, plasma cell levels are paradoxically increased (figure 3) ${ }^{16}$ IgG and IgA levels are transiently increased in adult asymptomatic patients and those with mild disease. ${ }^{16}$ Adults with severe COVID-19 have persistently raised IgG and IgA levels. This may correspond with higher neutralising antibodies, but higher IgG and IgA levels may also perpetuate innate cell activity, primarily in neutrophils expressing surface Fc receptors. ${ }^{26}$ Early phase rise in IgM levels was seen in asymptomatic, mild and severe cases. ${ }^{16}$ Early phase rise in IgM, persistent IgG and waning IgA levels were observed in paediatric COVID-19. ${ }^{1617}$ 


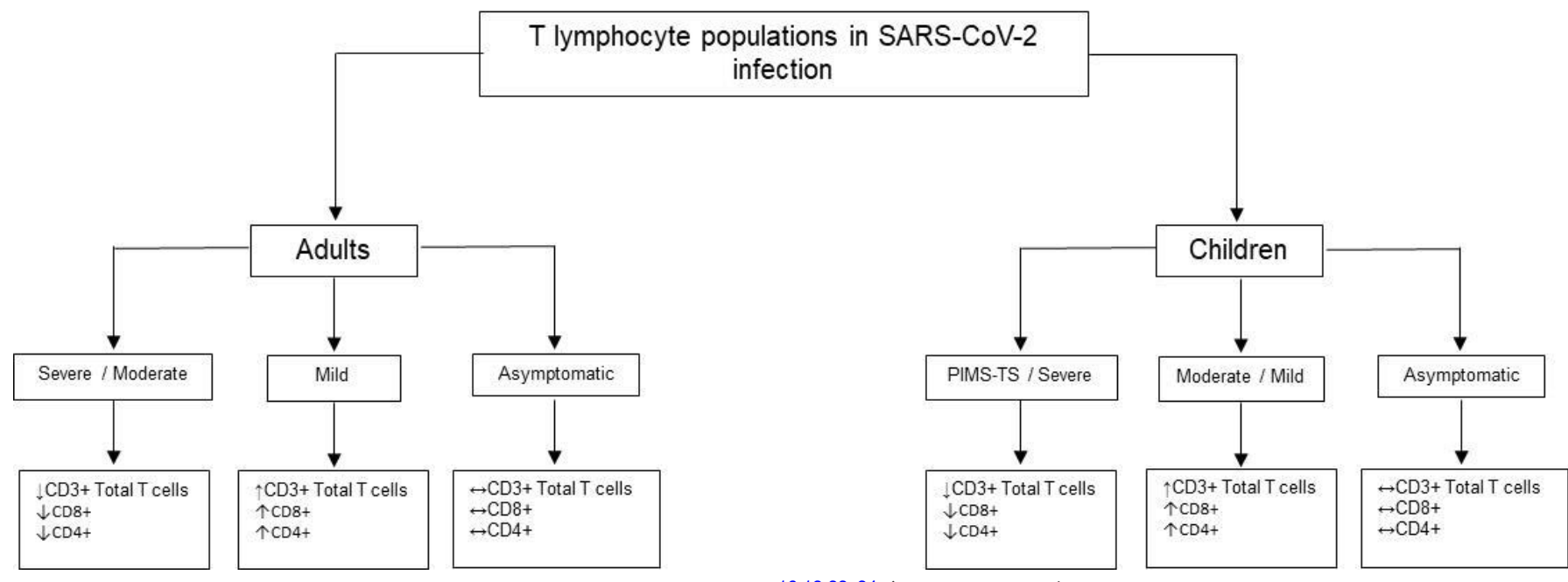

Figure 2 T lymphocyte populations in SARS-CoV-2 infection. ${ }^{16} 1922-24 \uparrow$, increased; $\downarrow$, decreased; $\leftrightarrow$, unchanged. CD, cluster of differentiation; PIMS-TS, paediatric multisystem inflammatory syndrome temporally associated with SARS-CoV-2.

The complement system is activated by the IgG and IgA SARS-CoV-2 immunocomplexes via the classical pathway as well as the Mannose-bindinglectin (MBL) pathway which senses the Spike glycoprotein. Complement activation ultimately leads to the membrane attackcomplex (C5b-9) but it also is a strong inducer of phagocytosis and NETS formation.

In contrast, no IgM was detected in children with PIMTS. ${ }^{27}$ These children were also SARS-CoV-2 PCR-negative, signifying that PIMS-TS is a clinical syndrome which occurs weeks following the acute infection. ${ }^{27}$ Children with PIMS-TS maintained high IgG levels with enduring Fc receptor-binding capacity which were capable of monocyte activation and sustained hyperinflammatory activity. ${ }^{26}$

Lastly, a study on hospitalised adults and children with COVID-19 in New York added to the cumulative evidence that mild disease seen in children was unlikely owed solely to adaptive immune responses; adults with severe COVID-19 mounted strong $\mathrm{T}$ cell responses and had higher neutralising antibodies compared with children with COVID-19 and/or PIMS-TS. ${ }^{28}$

\section{Anamnestic responses}

The course of severe adult COVID-19 is relatively slow (median 19 days post symptom onset for fatal $\operatorname{cases}^{2829}$ ), suggesting the protective involvement of memory B cells and T cells takes days to develop. ${ }^{29}$ Virus-specific memory CD4+ andCD8+ Tcell responses persist for at least 6 months, whereas virus-specific antibody responses declined after 3 months of infection. ${ }^{30}$ Moreover, SARSCoV-2-specific memory B cell responses evolved between 1.3 and 6.2 months after infection in a manner that was consistent with antigen persistence; viral antigens were detectable in enterocytes from adult intestinal biopsies even months after infection. ${ }^{31}$

Immune responses shortly after resolution of infection are not predictive of long-term memory in adults. ${ }^{31}$ Learning from SARS-CoV-1 and MERS, it is likely that few antibodies may persist for 2-3 years after infection, but $\mathrm{T}$

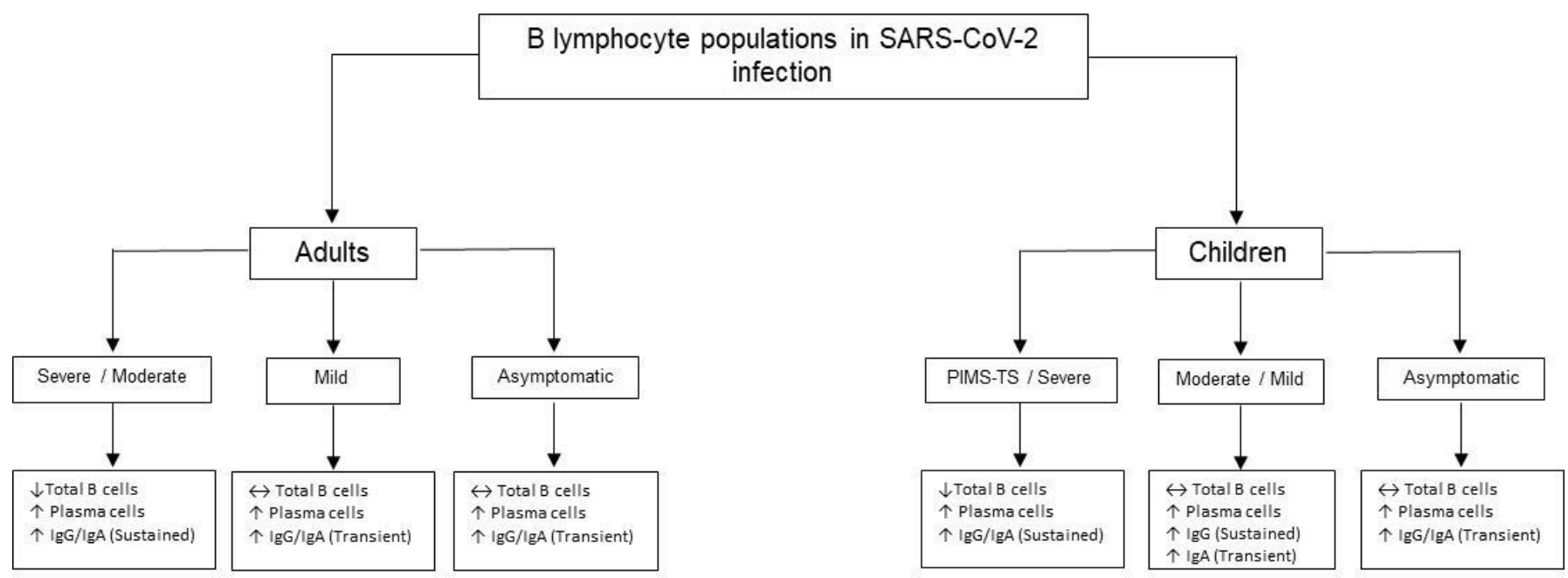

Figure 3 B lymphocyte populations in SARS-CoV-2 infection. ${ }^{15} 162526 \uparrow$, increased; $\downarrow$, decreased; $\leftrightarrow$, unchanged. PIMS-TS, paediatric multisystem inflammatory syndrome temporally associated with SARS-CoV-2. 
Table 2 Chemokine receptor expression in children and adults with SARS-CoV-2 infection 222732

\begin{tabular}{|c|c|c|c|c|c|c|c|}
\hline & \multicolumn{3}{|l|}{ Adults } & \multicolumn{4}{|l|}{ Children } \\
\hline & Severe & Mild & Asymptomatic & PIMS-TS & Severe & Mild & Asymptomatic \\
\hline CHEMOKINE & $\uparrow C X C L 10$ & $\uparrow C X C L 10$ & $\leftrightarrow$ CXCL10 & 个CXCL10 & $\leftrightarrow$ CXCL10 & $\leftrightarrow$ CXCL10 & $\leftrightarrow$ CXCL10 \\
\hline \multirow[t]{2}{*}{ RECEPTOR } & $\uparrow$ CXCL8 & $\uparrow C X C L 8$ & $\leftrightarrow$ CXCL8 & $\mathrm{n} / \mathrm{a}$ & $\leftrightarrow$ CXCL8 & $\leftrightarrow$ CXCL8 & $\leftrightarrow$ CXCL8 \\
\hline & 个CCL2 & 个CCL2 & $\leftrightarrow \mathrm{CCL} 2$ & $\leftrightarrow \mathrm{CCL} 2$ & $\leftrightarrow \mathrm{CCL} 2$ & $\leftrightarrow \mathrm{CCL} 2$ & $\leftrightarrow \mathrm{CCL} 2$ \\
\hline
\end{tabular}

$\uparrow$, increased; $\downarrow$, decreased; $\leftrightarrow$, unchanged.

CCL, CC chemokine ligand; CXCL, chemokine (C-X-C motif) ligand; n/a, not available; PIMS-TS, paediatric multisystem inflammatory syndrome temporally associated with SARS-CoV-2.

cell memory responses may last longer. Indeed, 28\%-50\% of adults not previously exposed to SARS-CoV-2 infection had cross-reactive $\mathrm{T}$ cells, likely mounted against other members of the coronavirus family that includes the common cold coronaviruses HCoV-OC43, HCoV-HKU1, HCoV-229E and HCoV-NL63. ${ }^{32}$ Children may have cross-protective antibodies against SARS-CoV-2 from the seasonal coronaviruses which commonly infect children. ${ }^{126}$

Immunosenescence, the natural decline in innate and adaptive immune cell function occurring with age, is most pronounced in elderly patients $>80$ years old. ${ }^{2} 617$ Immunosenescence may therefore predispose patients to poor viral clearance, promoting COVID-19 progression.

\section{Cytokine storm}

The hyperinflammatory response known as 'cytokine storm' occurs in the second week in severe and lifethreatening adult COVID-19; higher circulating proinflammatory cytokines are observed and clinically patients develop ARDS, multiorgan failure and disseminated intravascular coagulation (DIC). ${ }^{34}$ In ARDS, the cytokine storm is preceded by pulmonary infiltration of macrophages and neutrophils. ${ }^{34}$ These innate cells secrete powerful inflammatory cytokines including interleukin 6 (IL-6), IL-12, IL-10, IFN- $\gamma$ and tumour necrosis factor alpha (TNF- $\alpha$ ) and with varying reports of raised IL-1 $\beta$, IL-7, IL-8, IL-17 and G-CSF (granulocyte-colony stimulating factor). ${ }^{21} 33$ Levels of IL-6 and IL-10 along with $\mathrm{C}$ reactive protein (CRP) are considered prognostic markers (table 1) ${ }^{21}{ }^{33}$ Lymphopaenia, raised ferritin and D-dimer levels also correspond with disease severity in adult COVID-19. ${ }^{4}$

These clinical manifestations are rare in paediatric COVID-19; of children who develop severe illness, Twothirds will be due to PIMS-TS. ${ }^{7}$ PIMS-TS presents with a wide array of clinical features and variable disease severity, with shock, multiorgan failure, left ventricular impairment and coronary artery abnormalities described among them. ${ }^{34}$ The underlying cytokine storm in PIMS-TS is referred to as the macrophage activation syndrome (MAS). ${ }^{27}{ }^{34}$ MAS is encountered infrequently with other childhood infections, for example, EpsteinBarr virus, with medications and as a complication of autoimmune diseases. ${ }^{6} 1517$

Pierce and colleagues ${ }^{28}$ observed patients with PIMS-TS to have higher IL-6, IFN- $\gamma$, TNF- $\alpha$ and CXCL10 (chemokine (C-X-C motif) ligand) compared with acute paediatric COVID-19. The chemokines CXCL10 (also known as Interferon gamma-induced protein 10 (IP-10)), CXCL8 and CCL2 (CC chemokine ligand) (also known as Monocyte chemoattractant protein-1 (MCP-1)) are likewise detected in large amounts in adult COVID-19 (table 2). ${ }^{33}$ Other studies found levels of IL-6, IFN- $\gamma$ and TNF- $\alpha$ to be unchanged in paediatric COVID-19. ${ }^{23}$

In terms of laboratory biomarkers, patients with PIMSTS, similar to severe adult COVID-19, have higher CRP,

Table 1 Cytokine production in children and adults with SARS-CoV-2 infection 20263233

\begin{tabular}{|c|c|c|c|c|c|c|c|}
\hline & \multicolumn{3}{|l|}{ Adults } & \multicolumn{4}{|l|}{ Children } \\
\hline & Severe & Mild & Asymptomatic & PIMS-TS & Severe & Mild & Asymptomatic \\
\hline \multirow{7}{*}{ CYTOKINES } & 个IL-6 & $\uparrow I L-6$ & $\leftrightarrow \mid \mathrm{L}-6$ & $\uparrow I L-6$ & $\leftrightarrow \mid \mathrm{L}-6$ & $\leftrightarrow \mid \mathrm{L}-6$ & $\leftrightarrow \mid \mathrm{L}-6$ \\
\hline & $\uparrow I L-10$ & 个IL-10 & $\uparrow \mathrm{IL}-10$ & $\uparrow \mathrm{IL}-10$ & $\uparrow \mathrm{IL}-10$ & $\uparrow \mathrm{IL}-10$ & $\uparrow \mathrm{IL}-10$ \\
\hline & 个IFN & $\uparrow$ IFN & $\leftrightarrow \mathrm{IFN}$ & 个IFN & $\uparrow / \leftrightarrow \mathrm{FN}$ & $\uparrow / \leftrightarrow \mathrm{FN}$ & $\leftrightarrow \mathrm{IFN}$ \\
\hline & $\uparrow \mathrm{TNF} \alpha$ & $\uparrow \mathrm{TNF} \alpha$ & $\leftrightarrow \mathrm{TNF} \alpha$ & $\uparrow \mathrm{TNF} \alpha$ & $\leftrightarrow \mathrm{TNF} \alpha$ & $\leftrightarrow \mathrm{TNF} \alpha$ & $\leftrightarrow \mathrm{TNF} \alpha$ \\
\hline & $\uparrow / \leftrightarrow I L-1 \beta$ & $\uparrow / \leftrightarrow I L-1 \beta$ & $\leftrightarrow \mathrm{IL}-1 \beta$ & $\mathrm{n} / \mathrm{a}$ & $\leftrightarrow \mathrm{IL}-1 \beta$ & $\leftrightarrow \mathrm{IL}-1 \beta$ & $\leftrightarrow \mathrm{IL}-1 \beta$ \\
\hline & $\uparrow / \leftrightarrow \mathrm{IL}-8$ & $\uparrow / \leftrightarrow I L-8$ & $\leftrightarrow \mathrm{IL}-8$ & $\uparrow \mathrm{IL}-8$ & $\uparrow \mathrm{IL}-8$ & $\leftrightarrow \mathrm{IL}-8$ & $\leftrightarrow \mathrm{IL}-8$ \\
\hline & $\uparrow / \leftrightarrow \mathrm{IL}-17$ & $\uparrow / \leftrightarrow \mathrm{IL}-17$ & $\leftrightarrow \mathrm{IL}-17$ & $\uparrow \mathrm{IL}-17$ & $\uparrow / \leftrightarrow \mathrm{IL}-17$ & $\uparrow / \leftrightarrow \mathrm{IL}-17$ & $\leftrightarrow \mathrm{IL}-17$ \\
\hline
\end{tabular}

$\uparrow$, increased; $\downarrow$, decreased; $\leftrightarrow$, unchanged.

IFN, interferon; IL, interleukin; n/a, not available; PIMS-TS, paediatric multisystem inflammatory syndrome temporally associated with SARSCoV-2; TNF- $\alpha$, tumour necrosis factor alpha. 
ferritin and D-dimer levels, reflecting the inflammatory nature of the underlying pathology. ${ }^{7}$

These findings highlight the striking parallels in the immune responses and the biomarker levels observed between severe adult COVID-19 and PIMS-TS. The resulting end organ damage is, however, very different for these hyperinflammatory conditions.

PIMS-TS does not appear to be a direct result of SARS-CoV-2 infection but occurs following host immune attenuation after SARS-CoV-2 exposure. ${ }^{17}$ This concept is supported by the observation that PIMS-TS cases arose weeks following the peak of the first wave of COVID-19 and most children were SARS-CoV-2 PCR-negative and IgG-positive at the time of presentation. ${ }^{27}{ }^{34}$ In contrast, adult patients with COVID-19 may remain PCR-positive at the time of disease progression.

\section{ACE2 expression}

The expression of the SARS-CoV-2 entry receptor ACE2 has an almost Gaussian distribution, increasing from childhood into adulthood and declining with old age. ${ }^{125}$ It is found in most tissues but is highly expressed on type 2 pneumocytes. ${ }^{12}$ Evidence points towards a protective role, but the exact link between ACE2 and COVID-19 disease severity is unclear. ACE2 downregulation is seen in adults with comorbidities like hypertension and diabetes and also on SARS-CoV-2 spike protein binding. ${ }^{12}$ Animal studies suggest ACE2 protects against lung injury induced by SARS-CoV-2. ${ }^{25}$

Affinity for ACE2 may influence disease severity and trend; ACE2 in children are speculated to have a lower affinity to SARS-CoV-2 thus preventing viral entry into the host cell. ${ }^{5}$

TMPRSS2, transmembrane serine protease 2, helps to prime the spike glycoprotein and promotes viral entry. ${ }^{35}$ Lower TMPRSS2 levels are found in children and infants. These differences in ACE2 and TMPRSS2 expression may be pivotal in helping us understand the discrepancies in the clinical outcomes between children and adults.

\section{VIROLOGY AND VACCINES}

Since the announcement in December 2020 of a new COVID-19 strain in the UK, N501Y, multiple variants of concern have been identified. These include the E484K mutation first detected in South Africa, the P1. variant from Brazil and the double variant with both E484Q and L452R mutations identified in India. ${ }^{36}$ Worryingly these new strains are not only more infectious but are more virulent, causing greater morbidity and mortality ${ }^{36}{ }^{37}$ There is no evidence to date to establish children's susceptibility to the new variants, yet school closures in January 2021 in the UK were implemented to curb transmission of the more infectious strains.

With the SARS-CoV-2 vaccination programmes underway, there are serious concerns that mutations in the spike glycoprotein may render vaccine-induced antibodies ineffective or subneutralising. ${ }^{38}$ The current
COVID-19 vaccines are still considered to be effective against the N501Y variant but may be less so against the other new variants of concern. ${ }^{3738}$

Due to the global push to prevent mortality in high-risk groups, children are a low priority group for vaccination. Currently, the UK Joint Committee on Vaccination and Immunisation advises that only children from high-risk groups such as those with severe neurological disabilities who require residential care will be considered for vaccination (https://www.gov.uk/government/publications/ priority-groups-for-coronavirus-covid-19-vaccinationadvice-from-the-jcvi-30-december-2020).

The contribution children have on viral transmission and disease perpetuation in the community is uncertain. A rise in cases following the reopening of schools suggests the impact asymptomatic children have on transmission rates may be underestimated. This supports the argument that children are an important group to vaccinate. The USA saw a large reduction in rates of invasive pneumococcal disease in all ages caused by serotypes included in the pneumococcal vaccines PCV7 and PCV13 after routine use of these vaccines began for children (https://www.cdc.gov/vaccines/vpd/pneumo/public/ index.html). Sustained global decline in infection rates and control of the COVID-19 pandemic may not be achieved until children are included in the vaccination programmes.

\section{SUMMARY}

Striking clinical and immune differences exist between children and adult COVID-19. Children are largely asymptomatic and the dysregulated innate response is rarely described in paediatric COVID-19.

Conversely, severe adult COVID-19 and PIMS-TS appear to share similar immune signatures, yet their clinical response and end organ damage are distinctly different. The impact children have on viral transmission and disease perpetuation is uncertain; with the rise of cases in schools, including children in vaccination programmes will help curb the spread and control the pandemic.

\section{CONCLUSION}

The COVID-19 pandemic has highlighted gaps in our knowledge of viral immunology between adults and children. Deciphering the mechanism of children's resilience to disease could help target therapeutic interventions in adults and should be of high priority for future investigations.

Contributors SW: substantial contributions to the conception of the work; responsible for the body of the work, in drafting and revision of the manuscript; critical appraisal of the drafts/revision; agreement to be accountable for all aspects of the work. AR, DK: substantial contributions to the conception and design of the work; critical appraisal of the drafts/revision; final approval of the version to be published; agreement to be accountable for all aspects of the work. ZS: contributed to drafting and revision of the manuscript; critical appraisal of the drafts/revision; 
final approval of the version to be published; agreement to be accountable for all aspects of the work.

Funding The authors have not declared a specific grant for this research from any funding agency in the public, commercial or not-for-profit sectors.

Competing interests None declared.

Patient and public involvement Patients and/or the public were not involved in the design, or conduct, or reporting, or dissemination plans of this research.

Patient consent for publication Not required.

Provenance and peer review Not commissioned; externally peer reviewed.

Data availability statement Data sharing not applicable as no datasets generated and/or analysed for this study. Data included are from open access repositories.

Open access This is an open access article distributed in accordance with the Creative Commons Attribution Non Commercial (CC BY-NC 4.0) license, which permits others to distribute, remix, adapt, build upon this work non-commercially, and license their derivative works on different terms, provided the original work is properly cited, appropriate credit is given, any changes made indicated, and the use is non-commercial. See: http://creativecommons.org/licenses/by-nc/4.0/.

ORCID iD

Suz Warner http://orcid.org/0000-0001-5896-7036

\section{REFERENCES}

1 Miri SM, Noorbakhsh F, Mohebbi SR, et al. Higher prevalence of asymptomatic or mild COVID-19 in children, claims and clues. $J$ Med Virol 2020;92:2257-9.

2 Dhochak N, Singhal T, Kabra SK, et al. Pathophysiology of COVID-19: why children fare better than adults? Indian J Pediatr 2020;87:537-46.

3 Zhu J, Ji P, Pang J, et al. Clinical characteristics of 3062 COVID-19 patients: a meta-analysis. J Med Virol 2020;92:1902-14.

4 Nanda A, Vura NVRK, Gravenstein S. COVID-19 in older adults. Aging Clin Exp Res 2020;32:1199-202.

5 Zimmermann P, Curtis N. Why is COVID-19 less severe in children? A review of the proposed mechanisms underlying the age-related difference in severity of SARS-CoV-2 infections. Arch Dis Child 2020:archdischild-2020-320338.

6 Felsenstein S, Hedrich CM. SARS-CoV-2 infections in children and young people. Clin Immunol 2020;220:108588.

7 Yasuhara J, Kuno T, Takagi $\mathrm{H}$, et al. Clinical characteristics of COVID-19 in children: a systematic review. Pediatr Pulmonol 2020;55:2565-75.

8 Araújo LAde, Veloso CF, Souza MdeC, et al. The potential impact of the COVID-19 pandemic on child growth and development: a systematic review. J Pediatr 2020. doi:10.1016/j.jped.2020.08.008. [Epub ahead of print: 23 Sep 2020].

9 Ashikkali L, Carroll W, Johnson C. The indirect impact of COVID-19 on child health. Paediatr Child Health 2020;30:430-7.

10 Lotfi M, Hamblin MR, Rezaei N. COVID-19: transmission, prevention, and potential therapeutic opportunities. Clin Chim Acta 2020:508:254-66

11 Rajmil L. Role of children in the transmission of the COVID-19 pandemic: a rapid scoping review. BMJ Paediatr Open 2020:4:e000722.

12 Bhuiyan MU, Stiboy E, Hassan MZ, et al. Epidemiology of COVID-19 infection in young children under five years: a systematic review and meta-analysis. Vaccine 2021;39:667-77

13 Iwasaki A, Medzhitov R. Control of adaptive immunity by the innate immune system. Nat Immunol 2015;16:343-53.

14 Aboudounya MM, Heads RJ. COVID-19 and Toll-like receptor 4 (TLR4): SARS-CoV-2 may bind and activate TLR4 to increase ACE2 expression, facilitating entry and causing Hyperinflammation. Mediators Inflamm 2021;2021:1-18.

15 Wieczorek M, Abualrous ET, Sticht J, et al. Major histocompatibility complex (MHC) class I and MHC class II proteins: conformational plasticity in antigen presentation. Front Immunol 2017;8:292.
16 Carsetti R, Zaffina S, Piano Mortari E, et al. Different innate and adaptive immune responses to SARS-CoV-2 infection of asymptomatic, mild, and severe cases. Front Immunol 2020;11:610300.

17 Bordallo B, Bellas M, Cortez AF, et al. Severe COVID-19: what have we learned with the immunopathogenesis? Adv Rheumatol 2020;60:50.

18 Gkoutzourelas A, Bogdanos DP, Sakkas LI. Kawasaki disease and COVID-19. Mediterr J Rheumatol 2020;31(Suppl 2):268-74

19 Bordoni V, Sacchi A, Cimini E, et al. An inflammatory profile correlates with decreased frequency of cytotoxic cells in coronavirus disease 2019. Clin Infect Dis 2020;71:2272-5.

20 Gruber CN, Patel RS, Trachtman R, et al. Mapping systemic inflammation and antibody responses in multisystem inflammatory syndrome in children (MIS-C). Cell 2020;183:982-95.

21 Balasubramanian S, Rao NM, Goenka A, et al. Coronavirus Disease 2019 (COVID-19) in Children - What We Know So Far and What We Do Not. Indian Pediatr $2020 ; ; 57: 435-42$.

22 Bastard P, Rosen LB, Zhang Q, et al. Autoantibodies against type I IFNs in patients with life-threatening COVID-19. Science 2020;370:eabd4585.

$23 \mathrm{Li} \mathrm{H}$, Chen K, Liu M, et al. The profile of peripheral blood lymphocyte subsets and serum cytokines in children with 2019 novel coronavirus pneumonia. J Infect 2020;81:115-20.

24 Jacques FH, Apedaile E. Immunopathogenesis of COVID-19: summary and possible interventions. Front Immunol 2020;11:564925

$25 \mathrm{Wu} \mathrm{H}$, Zhu H, Yuan C, et al. Clinical and immune features of hospitalized pediatric patients with coronavirus disease 2019 (COVID-19) in Wuhan, China. JAMA Netw Open 2020;3:e2010895.

26 Bartsch YC, Wang C, Zohar T, et al. Humoral signatures of protective and pathological SARS-CoV-2 infection in children. Nat Med 2021;27:454-62

27 Perez-Toledo M, Faustini SE, Jossi SE, et al. Serology confirms SARS-CoV-2 infection in PCR-negative children presenting with paediatric inflammatory multi-system syndrome. medRxiv 2020. doi: 10.1101/2020.06.05.20123117. [Epub ahead of print: 07 Jun 2020].

28 Pierce CA, Preston-Hurlburt P, Dai Y, et al. Immune responses to SARS-CoV-2 infection in hospitalized pediatric and adult patients. Sci Trans/ Med 2020;12. doi:10.1126/scitranslmed.abd5487. [Epub ahead of print: 07102020$]$.

29 Zhou F, Yu T, Du R, et al. Clinical course and risk factors for mortality of adult inpatients with COVID-19 in Wuhan, China: a retrospective cohort study. Lancet 2020;395:1054-62. Erratum in: Lancet. 2020 Mar 28;395(10229):1038. Erratum in: Lancet. 2020 Mar 28;395(10229):1038.

30 Sette A, Crotty S. Adaptive immunity to SARS-CoV-2 and COVID-19. Cell 2021:184:861-80.

31 Gaebler C, Wang Z, Lorenzi JCC, et al. Evolution of antibody immunity to SARS-CoV-2. Nature 2021:591:639-44.

32 Grifoni A, Weiskopf D, Ramirez SI, et al. Targets of T cell responses to SARS-CoV-2 coronavirus in humans with COVID-19 disease and unexposed individuals. Cell 2020;181:1489-501. e15.

$33 \mathrm{Han} \mathrm{H}, \mathrm{Ma}$ Q, Li C, et al. Profiling serum cytokines in COVID-19 patients reveals IL-6 and IL-10 are disease severity predictors. Emerg Microbes Infect 2020;9:1123-30.

34 Ramcharan T, Nolan O, Lai CY, et al. Paediatric inflammatory multisystem syndrome: temporally associated with SARSCoV-2 (PIMS-TS): cardiac features, management and short-term outcomes at a UK tertiary paediatric Hospital. Pediatr Cardiol 2020;41:1391-401.

35 Schuler BA, Habermann AC, Plosa EJ, et al. Age-determined expression of priming protease TMPRSS2 and localization of SARSCoV-2 in lung epithelium. J Clin Invest 2021:131:e140766.

36 Faria NR, Mellan TA, Whittaker C. Genomics and epidemiology of a novel SARS-CoV-2 lineage in Manaus, Brazil. medRxiv.

37 Tang JW, Toovey OTR, Harvey KN, et al. Introduction of the South African SARS-CoV-2 variant 501Y.V2 into the UK. J Infect 2021;82:e8-10.

38 Andreano E, Piccini G, Licastro D, et al. SARS-CoV-2 escape in vitro from a highly neutralizing COVID-19 convalescent plasma. bioRxiv 2020. doi:10.1101/2020.12.28.424451. [Epub ahead of print: 28 Dec 2020]. 